\title{
Preliminarily Results from CARPET charge particles detector located in high cut off rigidity
}

\author{
Maghrabi A. ${ }^{1 *}$, Makhmutov V. ${ }^{2}$, Aldosari A. ${ }^{1}$, Almutairi M. ${ }^{1}$, Altilasi M. ${ }^{1}$, Philippov \\ M. ${ }^{2}$, Kalinin E. ${ }^{2}$, Alshehri $A^{1}$., Alfadhel $\mathbf{R}^{3}$., Almshari $\mathrm{B}^{3}$., 3Alrashied $\mathrm{J}^{3}$., Almutairi \\ $\mathrm{E}^{3}$., Alrashide $\mathrm{B}^{3}$.
}

1 National Centre For Applied Physics, King Abdulaziz City For Science and Technology, P.O. Box 6086 Riyadh 11442, Saudi Arabia.

2 Lebedev Physical Institute RAS, Moscow, Russia

3 Prince Nora Bin Abdulaziz University Address, Riyadh 844428, Saudi Arabia

E-mail: Email:amaghrabi@kacst.edu.sa

\begin{abstract}
Since their discoveries different types of radiation detectors, sensitive to various components of cosmic rays (CRs) have been developed to monitor and study their variations. As part of the King Abdulaziz City for Science and Technology (KACST) space research program and radiation detector laboratory activities, a CARPET detector was installed at KACST central Saudi Arabia (Riyadh; lat. 24 43; long. 46 40; alt. $613 \mathrm{~m} ; \mathrm{R}_{\mathbf{c}}=14.4 \mathrm{GV}$ ) for monitoring and exploring the variations of the CRs and their possible correlations with environment variables such as thunderstorms. The detector was designed and built by the Lebedev Physical Institute of the Russian Academy of Sciences (LPI, Moscow, Russia) within an international scientific cooperation between the LPI and the KACST and has been in operation since April 2016. In this paper, the technical aspects of this detector will be briefly discussed. Preliminary results obtained from this detector will be presented and discussed.
\end{abstract}

36th International Cosmic Ray Conference -ICRC2019-

July 24th - August 1st, 2019

Madison, WI, U.S.A.

\section{*A Maghrabi}




\section{Introduction}

Cosmic ray modulations have been studied using different types of detectors sensitive to various components of CRs. These detectors were placed at different locations around the world [1]. Riyadh, the capital of Saudi Arabia, has a unique location for monitoring and studying the variations of the CRs, due to its high geomagnetic cut off rigidity $\left(R_{c}=14.4 \mathrm{GV}\right)$. Since 2002, different types of $\mathrm{CR}$ detectors have been installed at this location. These include single channel muon detector [2], multi-wire detector [3], and neutron monitor [4]. Recently, we have installed and operated a CARPET charge particle detector. This detector was developed and designed by P. N. Lebedev Physical Institute, Russia, as part of international collaboration with King Abdulaziz City for Science and Technology (KACST), Saudi Arabia. CARPET detector is sensitive to the low energy component of the secondary CRs. The scientific goal of this instrument is the study of the CR flux modulation in different timescales and to better understand the correlations between the CR count rate and thunderstorm activity. In this paper, preliminary results obtained from the installed detector will be presented.

\section{The Detection System}

Fig. 1 is a schematic diagram shows the main components of the detection system.

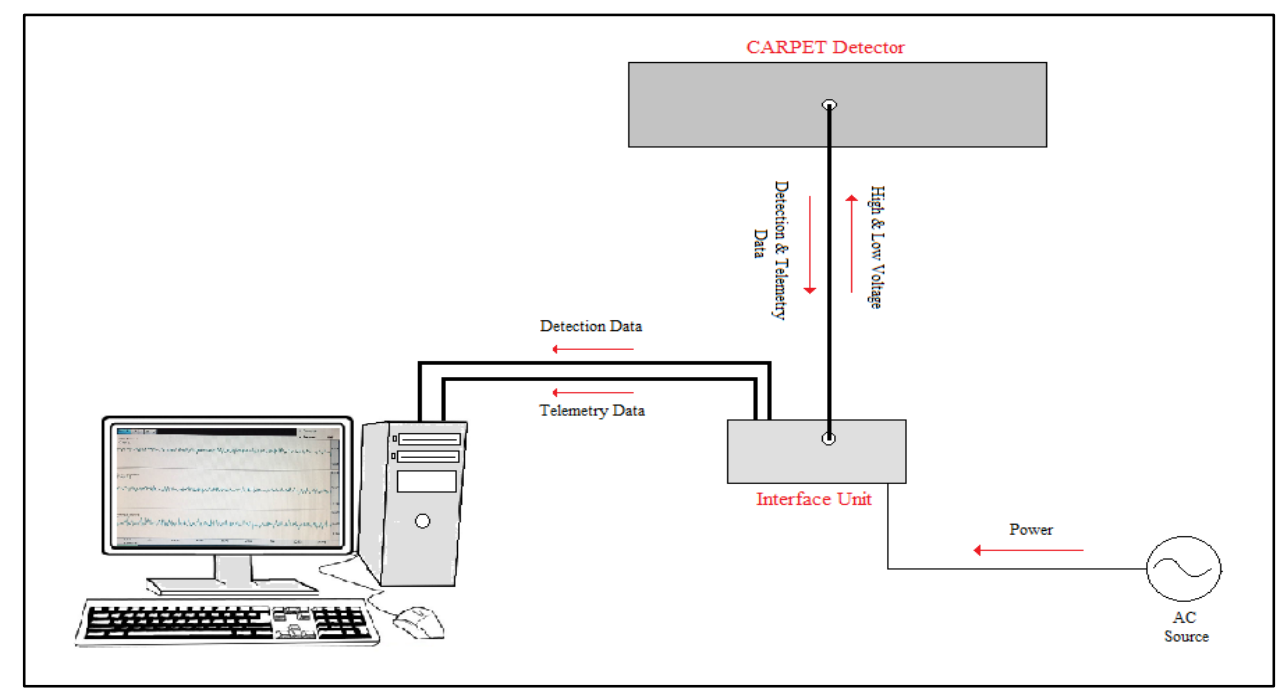

Fig. 1: schematic diagram for CARPET detector.

The detector consists of 120 counters grouped in 12 blocks of 10 Geiger counters located on a platform $1.5 \times 1.5 \mathrm{~m}^{2}$ in size. Each block consists of five upper and five lower counters separated by aluminum absorber with a thickness of $7 \mathrm{~mm}$ (Fig. 2). 


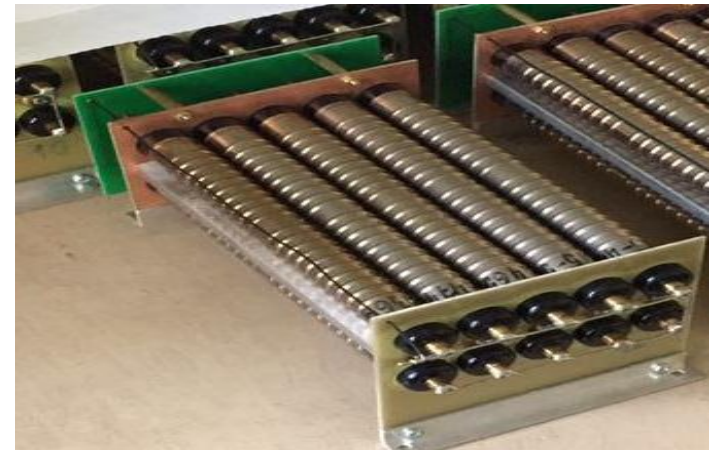

Fig. 2: Photo shows one block of CARPET detector.

The detector has three data channels: (I) UP channel records the charged particles that hit the 60 upper layer counters; (II) LOW channel records the charge particles counts from the 60 lower layer counters, and (III) TELESCOPE

(TEL) channel registers the total counts in coincidence between the upper and lower layers.

The UP and LOW channels are sensitive to electrons and positrons with energies above 0.2 $\mathrm{MeV}$, protons above $5 \mathrm{MeV}$ and photons with energy above $20 \mathrm{keV}$. On the other hand, the TEL detects mainly electrons with energies greater than $5 \mathrm{MeV}$, protons with energies greater than 30 $\mathrm{MeV}$, and muons with energies greater than $15.5 \mathrm{MeV}$. A data acquisition unit was developed for logging the data, interfacing with the electronics, and storing the data. The electronics make data records with $1 \mathrm{~ms}$ resolution. More details about this detector are given in several research papers [e.g., 5].

\section{Results and Discussions}

\subsection{CARPET Performance}

Two CARPET detectors were installed at KACST. The first located inside the radiation detector lab building and the second was installed at the roof of the building. Fig. 3 indicates the hourly variations of the CR charged particles recorded by the CARPET UP channel located inside the laboratory and the CARPET UP channel placed at the roof of the building. It is evident that the behavior from both detectors is comparable with each other. However, due to the absorbing materials above the lab, the CR counts from the detector at the roof are higher. For the purpose of this study, data from the CARPET detector placed inside the lab will be used.

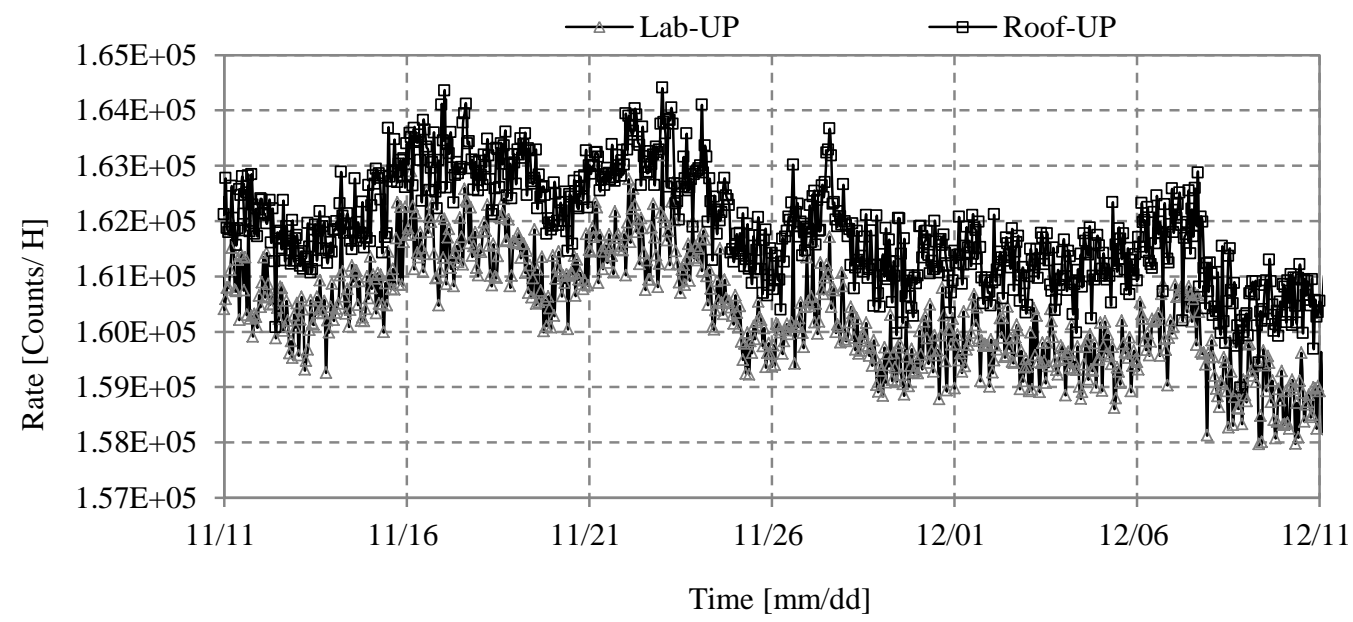

Fig.3: Hourly variations of the CRs detected by the CARPET's UP channels for the period between 11 November to 12 December 2017. 
Fig. 4 shows the hourly variations of the CR charged particles detected by the CARPET's three channels over a period of two months. Clearly seen, that the behavior of the three channels are the same, however, the counts are different.

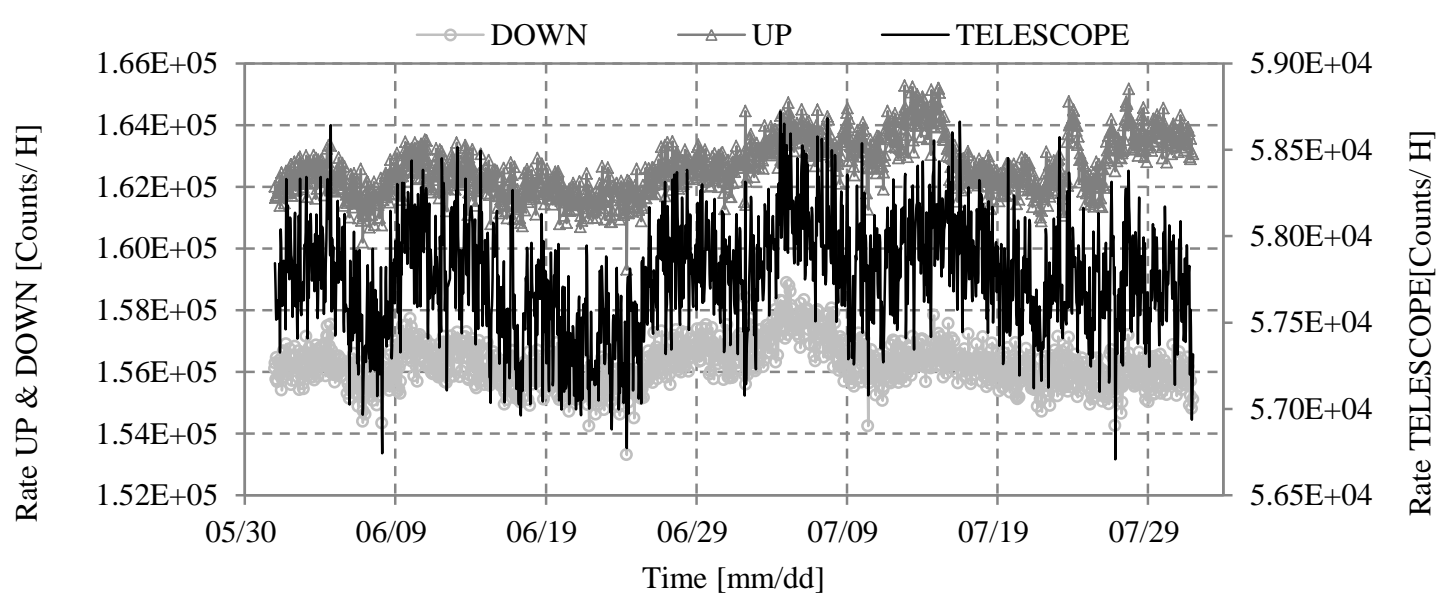

Fig.4: Hourly variations of the CR detected by the CARPET's three channels for the period between 1 June to 30 July 2018.

\subsection{Atmospheric effects}

Atmospheric pressure is considered as the most effective and important atmospheric variable affect the detected CRs at the ground level. It represents the atmospheric mass above the detector. To study the modulation of the primary $\mathrm{CR}$, it is important to remove the atmospheric pressure effect on the secondary CR particles [6,7]. Fig. 5 shows the variations of the hourly values of the atmospheric pressure and the CR particles detected by the CARPET telescope channel over a period of two months. It is evident that the muon count rate is anticorrelated with atmospheric pressure.

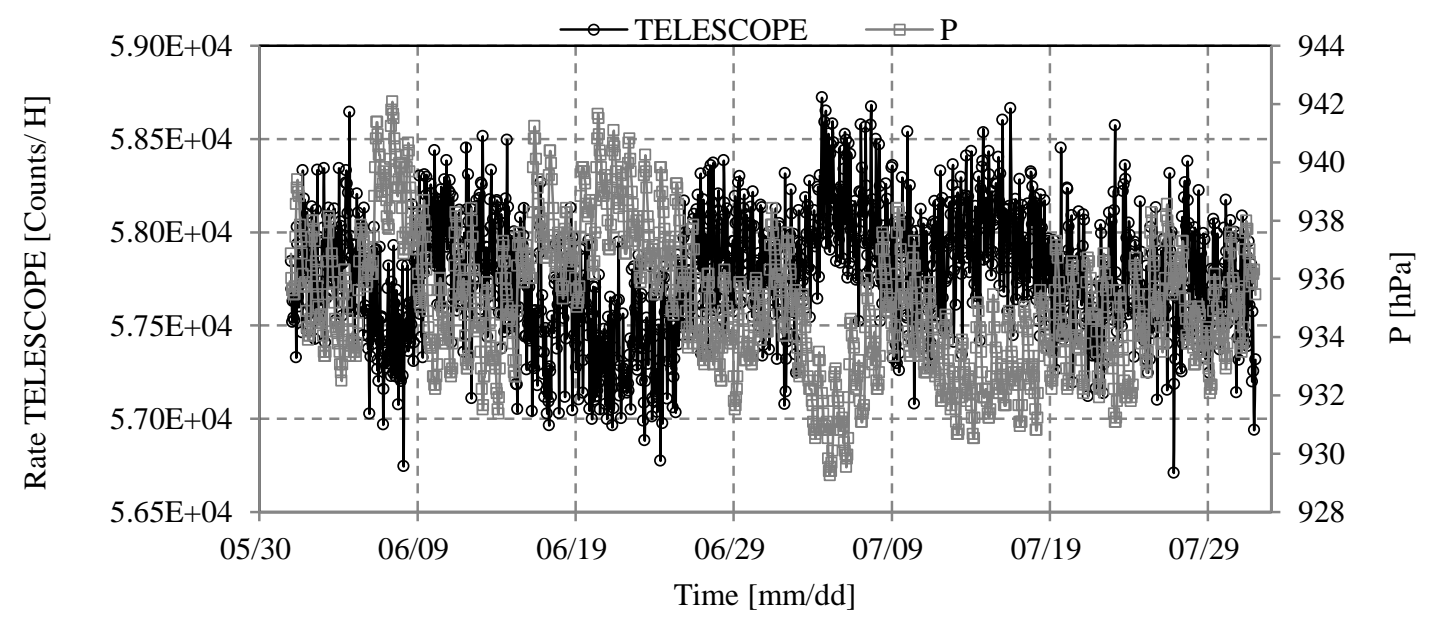

Fig. 5: Hourly variations of the atmospheric pressure $(P)$ and the CRs detected by the TEL channel 
between 1 June to 30 July 2018.

Correlation between the hourly values of the atmospheric pressure and the CR particles detected by the TEL channel is presented in Fig. 6. Using a least-squares fitting method, we found the barometric coefficient $\sim-0.12 \pm 0.01 \% / \mathrm{hPa}$ and a correlation coefficient $\sim 0.72$. This value is smaller than the obtained coefficient from the CARPET detector installed at the El Leoncito station (CASLEO, San Juan, Argentina, cutoff rigidity 11.8GV) [8]. This may be due to the differences in locations and the prevailing atmospheric conditions between the two sites.

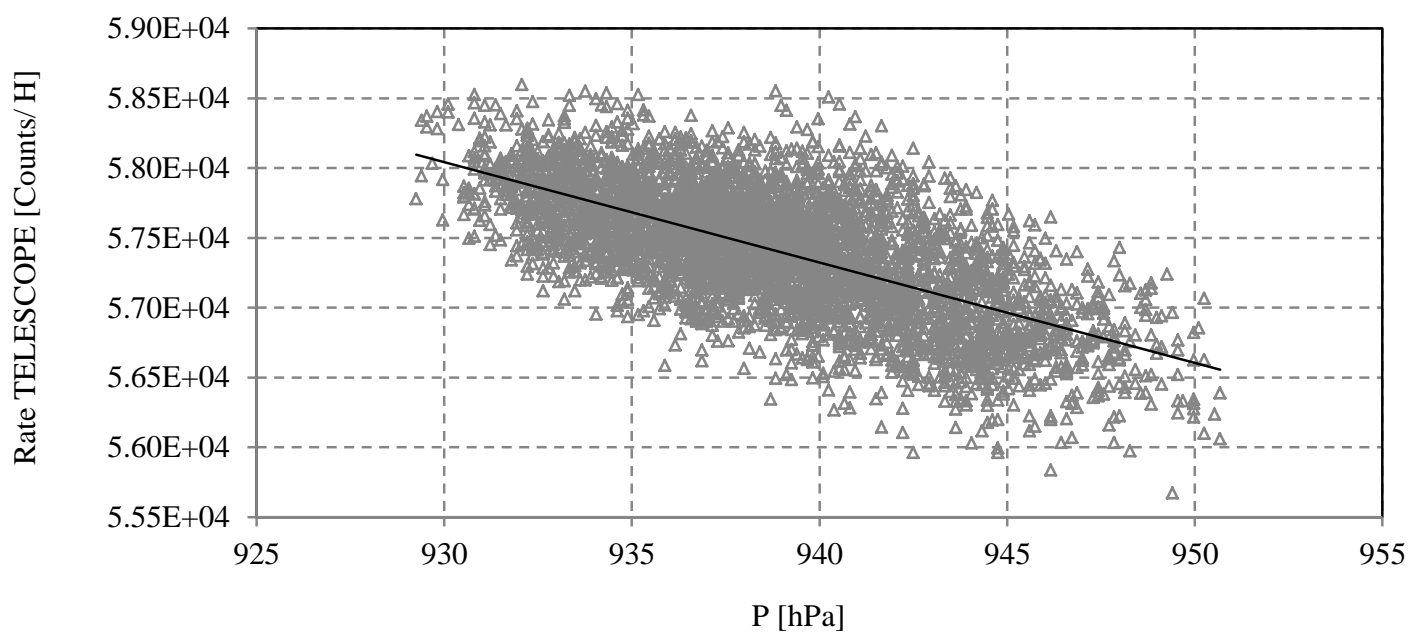

Fig. 6: Scatter plot between the atmospheric pressure and the CR particles detected by the TEL channel.

Fig.7 is plot showing the variations of the raw CR rate from the TELESCOPE channel and the pressure-corrected rate using the obtained coefficient. There are observable differences between the corrected and uncorrected rates, i.e. about $0.22 \%$ on average.

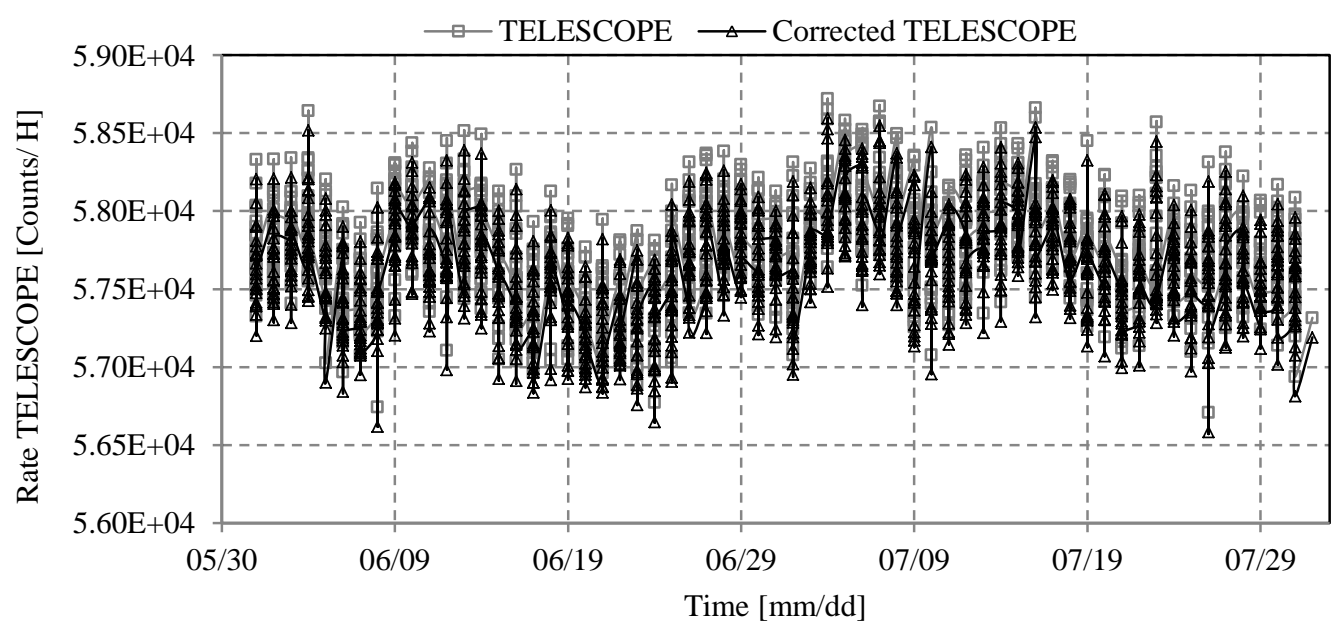

Fig. 7: Time series compares the raw $C R$ and the pressure-corrected CR data from the CARPET TEL 
channel between 1 June to 30 July 2018.

\subsection{CARPET vs. KACST muon detector}

The ability of CARPET detector to detect high-energy CR particles was compared with the existed KACST muon detector. This is a single channel scintillator detector installed at KACST and has been in operation since 2002 [2]. Fig.8 shows the hourly variations of the CARPET TEL channel data and data from the muon detector for the period between June to July 2018. It is obvious that the behavior of the CARPET recorded data follows that of the muon detector.

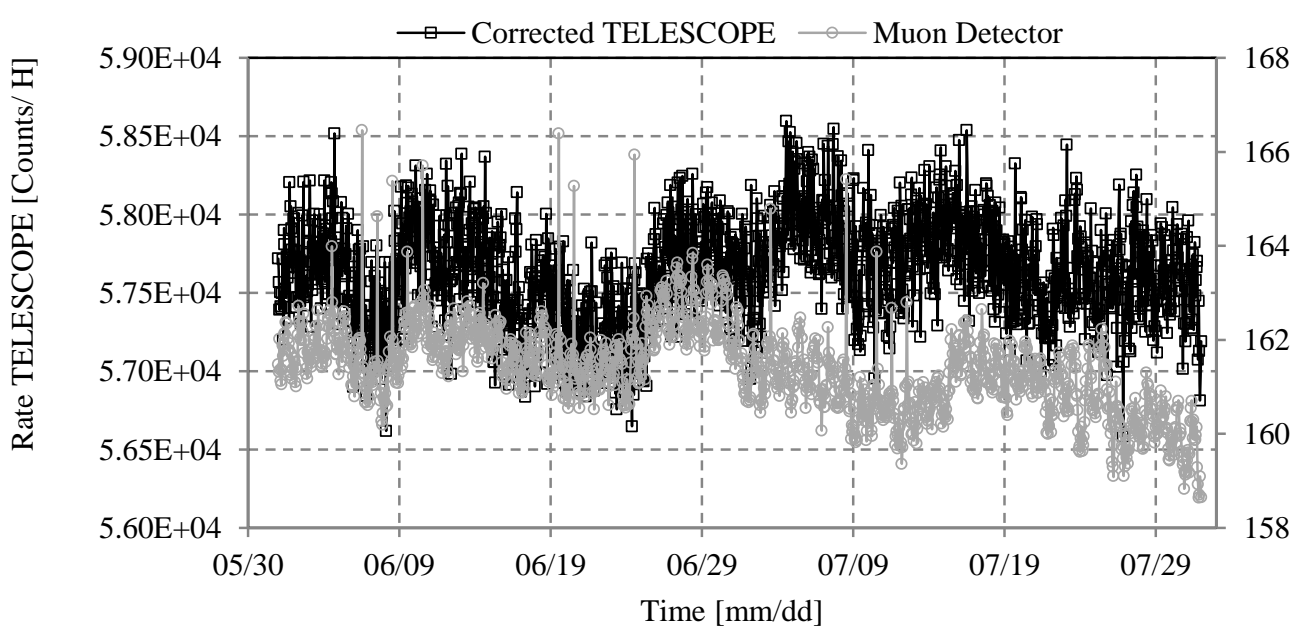

Fig. 8: Hourly variations of the CR data detected by the CARPET TEL and KACST $1 \mathrm{~m}^{2}$ scintillator detector.

\subsection{CR Variations during High Electric Field Activity- Case study.}

One of the main goals of installing CARPET detector is to study and understand the variations of the $\mathrm{CR}$ rate during some environmental disturbances such as, thunderstorm activities. For this purpose, continuous measurements of the atmospheric electric field at the KACST have been performed since January 2018 using the EFM 100 electric field monitor.

Since its installation in 2016, several events consisting of increases in the count rates have been detected by CARPET. Fig. 9 shows the 1minute resolution CR data from the CARPET two channels and the earth electric field during the period between 14-16 February 2019. During this period the EF values were intensified and there were corresponding observable increase in the rate of charge particles detected by CARPET. On the 14th around 19:35 the EF jumped from the background level to about $1.3 \mathrm{kV} / \mathrm{m}$. In response to this variation, the charge particles from the UP and DOWN channels increased by $4.7 \%$ and $4.5 \%$, respectively. The increase in the CR flux due to the atmospheric electric field variations has been previously reported [5,6,9-11]. Hence, future correlation analyses between the EF and charge particles changes observed by CARPET will allow us, to investigate and understand the relationship between the atmospheric effect and charge particles. 

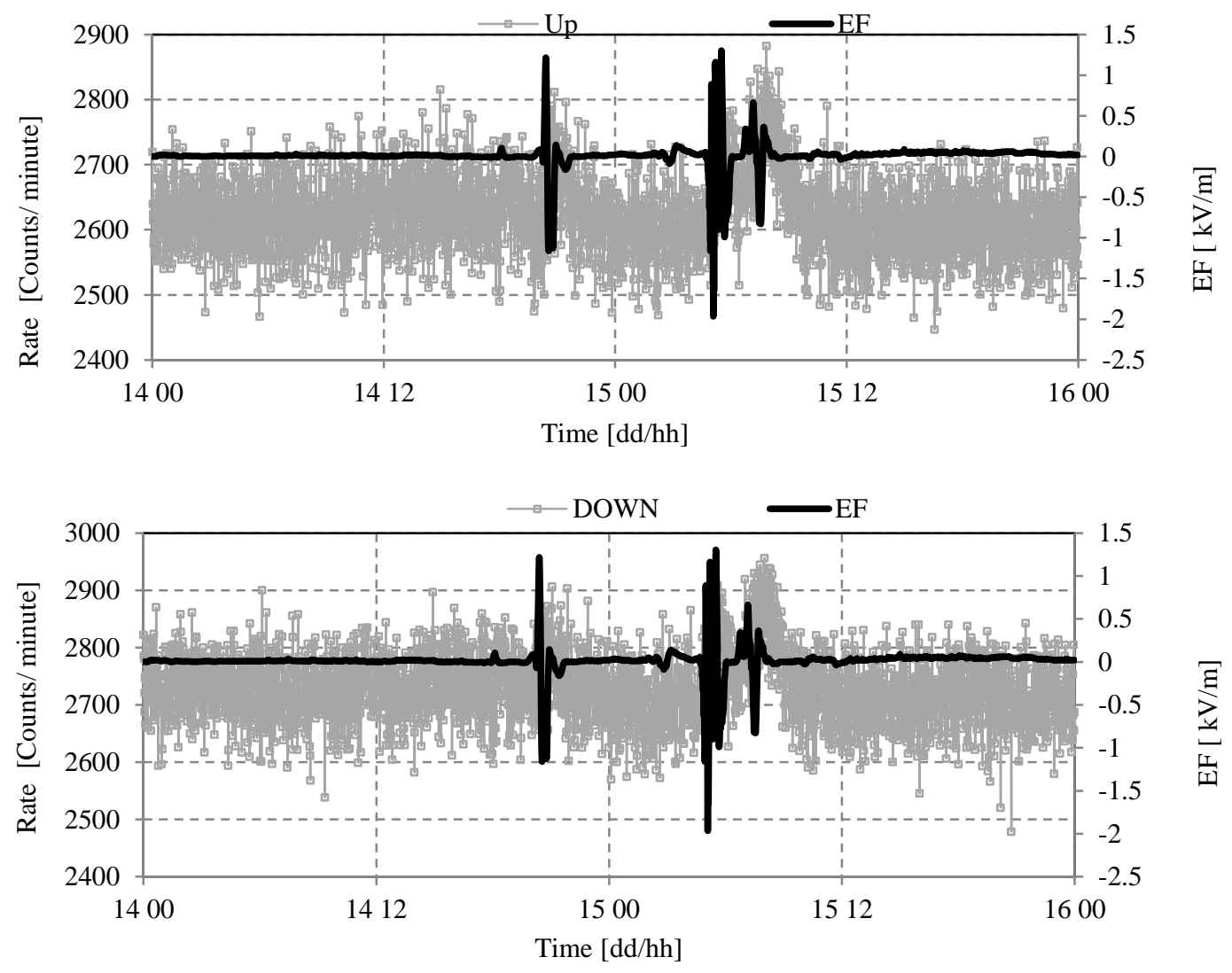

Fig. 9: Time series of the electric field and CR particles detected by CARPET UP and DOWN channels for the period between 14-16 February 2018.

\section{Conclusion}

In 2016 a CARPET cosmic ray detector was installed at the King Abdulaziz City for Science and Technology (KACST) in Riyadh, Saudi Arabia $(\mathrm{Rc} \approx 14.4 \mathrm{GV})$ to complements the previously installed detectors. The main goal of this detector is to record CR particles with lower energies than the existed detectors and study their variations and their relationship with atmospheric processes. Preliminary results obtained from this detector were presented and discussed.

\section{Acknowledgments}

The authors would like to thank King Abdulaziz City for Science and Technology (KACST) for supporting this work. 


\section{References}

[1] P. Stoker. 2009. The IGY and beyond: A brief history of ground-based cosmic-ray detectors, Advances in Space Research. 44, 1081-1095.

[2] A. Maghrabi et al., 2012. The KACST muon detector and its application to cosmic-ray variations studies, Advances in Space Research, 50, 6.

[3] A. Maghrabi et al., 2017. Small three-layer multiwire-based detector for cosmic ray muon variation studies at high geomagnetic rigidity cutoff; Journal of Astronomical Telescopes, Instruments, and Systems, 3(2).

[4] A. Maghrabi et al., 2019. Preliminarily Results from A Mini Neutron Monitor in Central Saudi Arabia, 36th International Cosmic Ray Conference -ICRC2019- Madison, WI, U.S.A.

[5] R. De Mendonça et al., 2011. Long-term and transient time variation of cosmic ray fluxes detected in Argentina by CARPET cosmic ray detector, J. Atmos. Sol.-Terr. Phys., 73.

[6] L. Dorman, 2004. Cosmic rays in the Earth's atmosphere and underground, Kluwer Academic Publishers, USA.

[7] A. Maghrabi et al., 2015. Influence of the Atmospheric Mass on the High Energy Cosmic Ray Muons during a Solar Cycle, Advances in Astronomy doi.org/10.1155/2015/939146.

[8] R. DeMendonça et al., 2013. Analysis of atmospheric pressure and temperature effects on cosmic ray measurements. J. Geophys. Res118, 1403.

[9] X. Zhou, et al., 2016. Effect of near-earth thunderstorms electric field on the intensity of ground cosmic ray positrons/electrons in Tibet. Astroparticle Physics, 84, 107-114.

[10] N. Khaerdinov and A. Lidvansky. 2013. Variations of cosmic ray muon flux during thunderstorms J. Phys.: Conf. Ser. 409012230

[11] A. Lidvansky. 2003. The effect of the electric field of the atmosphere on cosmic rays J. Phys. G: Nucl. Part. Phys. 29. 